Revue internationale P.M.E.

Économie et gestion de la petite et moyenne entreprise

Revue

internationale

PME

\title{
Pour une approche ethnométhodologique de la PME
}

\section{Jean-Michel Plane}

Volume 11, numéro 1, 1998

URI : https://id.erudit.org/iderudit/1009039ar

DOI : https://doi.org/10.7202/1009039ar

Aller au sommaire du numéro

Éditeur(s)

Presses de l’Université du Québec

ISSN

0776-5436 (imprimé)

1918-9699 (numérique)

Découvrir la revue

Citer cette note

Plane, J.-M. (1998). Pour une approche ethnométhodologique de la PME. Revue internationale P.M.E., 11(1), 123-140. https://doi.org/10.7202/1009039ar

\section{Résumé de l'article}

Cet article est consacré à la discussion de problèmes épistémologiques et méthodologiques posés par l'observation des pratiques de gestion au sein de PME. Dans cette perspective, les questions du contexte particulier d'intervention en PME, de la neutralité des intervenants en gestion et de l'inévitable dialectique immersion- distanciation vis-à-vis des objets étudiés sont discutées. L'objectif de l'article est de tenter de montrer l'intérêt théorique et surtout méthodologique d'une approche ethnométhodologique de la PME. 


\title{
Pour une approche ethnométhodologique de la PME
}

Jean-Michel PLANE

Université Paul-Valéry, Montpellier III

MOTS-CLES

\section{Épistémologie - Constructiviste - Ethnométhodologie Recherche-intervention - Management - PME Méthodologie qualitative de la recherche - Système de gestion}

\begin{abstract}
RÉSUMÉ
Cet article est consacré à la discussion de problèmes épistémologiques et méthodologiques posés par l'observation des pratiques de gestion au sein de PME. Dans cette perspective, les questions du contexte particulier d'intervention en PME, de la neutralité des intervenants en gestion et de l'inévitable dialectique immersiondistanciation vis-à-vis des objets étudiés sont discutées. L'objectif de l'article est de tenter de montrer l'intérêt théorique et surtout méthodologique d'une approche ethnométhodologique de la PME.
\end{abstract}

\section{L'AUTEUR}

Jean-Michel Plane est maître de conférences en sciences de gestion à l'Université Paul-Valéry, Montpellier III. II participe aux travaux scientifiques de l'ERFI de l'Université de Montpellier I. Ses préoccupations de recherche portent actuellement sur les méthodologies qualitatives de la recherche en PME ainsi que sur les épistémologies constructivistes. II exerce également des activités de conseil auprès de diverses organisations et enseigne principalement la théorie des organisations et le management des ressources humaines. Il est également responsable des enseignements de méthodologie de l'intervention en management au sein du DESS de gestion stratégique des ressources humaines de l'Université de Montpellier III. Adresse: Route de Mende-B.P. 5043-34032 Montpellier Cedex 1-France. L'auteur tient à remercier son collègue Olivier Torrès pour ses observations et ses suggestions contribuant ainsi à l'amélioration de la version initiale. 


\begin{abstract}
This article is devoted to the discussion of epistemologic and methodological problems set by the observation of the management practices within PME. From this point of view, are debated questions of the specific context concerning interventions inside the PME, of the contributor's neutrality about management and of the inescapable dialectic "immersion-distance" toward the examined objects. The purpose of the article attempts to point out the theoretic and mostly methodological interest of an ethnomethodological approach of the PME.
\end{abstract}

\title{
RESUMEN
}

Este articulo es destinado a la discusion de problemas epistemologicos y metodologicas sentados por la observacion de las practicas de gestion dentro de PME. En esta perspectiva, las cuestiones del contexto especifico de intervencion en PME, de la neutralidad de los interventores en gestion y del consabido dialectico immersion-distanciamiento enfrente de los objetivos estudiados estan controvertidas. El objetivo del articulo es de intentar de ensenar el interes teorico y sobre todo metodologico del enfoque ethnometodologica de la PME.

\section{Introduction}

Cette fin de siècle est incontestablement dominée par le dynamisme des petites et moyennes entreprises. En effet, force est de constater que leur contribution à la croissance économique des pays occidentaux est considérable même si les multinationales restent les « porte-avions » des PME à l'exportation pour reprendre l'expression récemment utilisée dans un article du journal Le Monde (Laronche, 1997). Autrement dit, de grandes entreprises aident les petites à trouver des débouchés à l'étranger. Par exemple, certaines PME bénéficient de l'expérience et du carnet d'adresses de la multinationale, ainsi que de ses connaissances en matière de lois et de réglementations nationales sur des investissements étrangers. Économie de temps, d'argent, d'énergie, ce partenariat entre PME et multinationales semble manifestement en plein essor. Le développement de la mondialisation de l'économie devrait renforcer cette tendance (Bizaguet, 1991). En effet, l'installation progressive de réseaux d'informations planétaires grâce aux progrès de la technologie et des services contribue à une nouvelle approche plus unifiée du marché mondial. Dans une telle perspective, il apparaît important de renforcer l'intelligence économique des PME en développant en leur sein de nouvelles compétences, par exemple, en gestion de l'information internationale ou encore en management interculturel et en marketing international. À partir de ces actions de formation relativement novatrices, on soutient l'idée que les PME ont particulièrement besoin de tels investissements immatériels contribuant ainsi à leur nouvelle productivité au sein d'une société informationnelle et d'une économie de savoir. C'est dans ce contexte que la démarche de recherche-intervention en management, fondée sur une analyse 
en profondeur du potentiel de l'entreprise et sur la mise en évidence de nouvelles perspectives d'actions, peut connaître un certain renouveau en PME dans le sens où les activités de ces firmes «s'immatérialisent». Par exemple, il s'agit d'être capable de mettre en marché puis de vendre une partie substantielle de la production à partir de l'Internet. En ce sens, elles ont aujourd'hui besoin de mieux percevoir les possibilités de développement économique offertes par le phénomène de globalisation et de renforcer leurs capacités d'actions commerciales. Elles semblent donc aujourd'hui plus ouvertes à l'implantation de méthodes et d'outils de management dans une logique d'accroissement de cette intelligence économique et de ce potentiel stratégique. Dans une logique de développement organisationnel, on avance la thèse de la nécessité d'une approche ethnométhodologique de la PME, fondée sur l'étude ethnographique des processus d'interactions entre des intervenants-chercheurs et les acteurs des PME et des transformations qui en résultent dans une perspective de performance. Ce travail de recherche vise à éclairer la nature et la complexité des processus d'interactions chercheurs-acteurs de même que leurs effets sur la performance économique et sociale. Dans cette optique, cette approche s'appuie sur des investigations effectuées à partir de situations réelles d'interactions avec de multiples acteurs rencontrés dans les organisations, objets de recherchesinterventions. L'objectif de cet article est de présenter, à partir de travaux cliniques, une approche ethnométhodologique de la PME ${ }^{1}$. Dans cette perspective, nous exposerons dans un premier temps des considérations théoriques et méthodologiques posées par l'ethnométhodologie et la recherche-action en PME. Dans un second temps, l'article mettra en relief la contribution de la démarche ethnométhodologique à l'analyse du fonctionnement des PME.

\section{Ethnométhodologie et recherche-action en PME}

\subsection{Ethnométhodologie et sciences de gestion}

Dans le cadre d'une recherche-action, le chercheur est un intervenant-chercheur, engagé dans un processus où il y a simultanément création de connaissance et changement (Plane, 1994 et 1996b). Dans cette optique, il semble que la participation active des acteurs des organisations à la recherche elle-même peut prendre deux formes :

1. Cet article s'appuie sur plusieurs recherches-interventions réalisées entre 1990 et 1997 au sein de diverses entreprises et organisations. Parmi celles-ci, on a privilégié les cas de PME. Précisément, notre base d'information est constituée par trois petites entreprises (moins de 10 salariés) : une entreprise de conseil en organisation, une société de production audiovisuelle, une entreprise spécialisée dans la production de composants électroniques ainsi qu'une association de réinsertion sociale (environ 30 salariés). 
- Dans certains cas, les acteurs le font sans le savoir dans la mesure où le chercheur utilise l'action qu'il pilote afin de produire des connaissances, mais ses interlocuteurs sur le terrain n'en sont pas conscients.

- Dans d'autres cas, les acteurs contribuent activement à la construction même du processus de recherche. À travers ce type de recherche-action, les acteurs des organisations sont considérés comme détenant, eux aussi, une part de la connaissance et ayant des vues pertinentes sur la problématique à élaborer. Cette conception est proche de ce que l'on appelle «l'ethnométhodologie » (Garfinkel, 1967). L'ethnométhodologie fait ainsi appel à un partenariat (Cargese-CNRS, 1991) entre les chercheurs et les acteurs des organisations (Yanat, 1992). Les recherches-interventions en management socio-économique (Savall, 1989) mises en œuvre depuis plusieurs années dans une très grande diversité d'organisations sont également assez proches de cette logique même si les chercheurs ont une approche plus déterministe puisqu'ils transfèrent, en les acclimatant, des concepts, des méthodes et des outils ainsi qu'une méthodologie d'intervention. En général, on constate que la question des méthodes employées dans le cadre des recherches-interventions en management stratégique revêt une importance fondamentale (Morin, 1987). Ces recherchesexpérimentations présentent de manière générale les caractéristiques suivantes. Au plan méthodologique, les relations entre les chercheurs et les acteurs de l'organisation se manifestent par de fortes interactions et conduisent à infléchir la recherche et à l'orienter vers d'autres directions plus fécondes que la problématique initiale. Cette option méthodologique semble constituer le propre de la démarche heuristique. Au plan épistémologique, la plupart des travaux sur la recherche-intervention (par exemple, l'école sociotechnique) indiquent que les chercheurs privilégient une conception phénoménologique de la recherche en étudiant les phénomènes observés à partir d'actions de changement mises en œuvre. Cette option épistémologique semble constituer le propre de la recherche transformative dans les organisations qui vise à une théorisation à partir d'observations réalisées à la suite d'actions de changement mises en œuvre. Progressivement dans ces interventions, les chercheurs ont privilégié, semble-t-il, le paradigme de l'interaction avec les acteurs qui les a conduits à une approche de recherche plus transformative de l'objet observé. Dans cette perspective, les chercheurs pratiquent la « recherche interactive» (Girin, 1986 , p. 170 et s.). Une recherche interactive présente ainsi quatre caractéristiques. En premier lieu, les connaissances élaborées sont fondées sur un travail de terrain. En second lieu, le travail de terrain s'ajuste aux faits et aux situations pouvant l'infléchir. Par ailleurs, les autochtones, c'est-à-dire les gens du terrain, ont un rôle important sur le déroulement de la recherche. Enfin, les intervenants-chercheurs sont responsables de l'interprétation des 
informations et des constructions théoriques qui en découlent. Cette option épistémologique revient à tenter d'apporter des éléments de réponse à la problématique centrale suivante : «Peut-on tirer des connaissances valides à partir de données subjectives recueillies à chaud ?» (Girin, 1986) Au plan épistémologique, compte tenu de cette problématique, la tentative de réponse apportée par plusieurs centres de recherche en sciences de gestion revient à noter qu'il est possible d'extraire des connaissances sur un terrain en les objectivant, c'est-à-dire en les rapportant aux situations dans lesquelles elles ont été produites. Il est remarquable de constater que certains chercheurs en sciences de gestion, comme par exemple Bouchikhi, ne considèrent pas les phénomènes de transfert et de contre-transfert, c'est-à-dire les projections faites par les acteurs de l'organisation sur les chercheurs et réciproquement, comme des phénomènes perturbateurs qu'il faut éliminer ou, pis, occulter. En effet, dès qu'il y a interaction, il y a nécessairement des attentes, des préférences, des tabous, des non-dits... dont «l'analyse peut être riche d'enseignements au regard du fonctionnement intime de l'organisation étudiée» (Bouchikhi, 1988, p. 23).

L'approche de Marchesnay (1985, p. 55) semble relativement proche de ces conceptions de la recherche en sciences de gestion dans la mesure où il identifie, au sein de ce qu'il appelle «le protocole de l'analyse» de la recherche, une phase préanalytique qui comprend l'analyse des éléments suivants :

- L'ensemble des sentiments, impulsions, etc., propres aux chercheurs en sciences sociales.

- L'ensemble des valeurs, des croyances, des attitudes, des normes sociétales auxquelles le chercheur se réfère dans son analyse. Celles-ci peuvent lui être propres, ou lui être dictées de fait par le paradigme auquel il se rattache.

- L'ensemble des informations et des valeurs implicitement contenues par les hypothèses fondamentales auxquelles le chercheur se rattache. Ce paradigme est de fait un «canaliseur de vision».

Autrement dit, l'auteur suggère d'expliciter, dans le cadre d'une phase préanalytique de la recherche, ces différents éléments le plus souvent implicites dans la formulation de ce protocole de l'analyse. Au total, on retiendra que cette phase fait partie intégrante de la démarche de modélisation en sciences de gestion. L'approche de la recherche en gestion développée par Savall (1985, p. 242) est fondée sur les éléments suivants. D'une part, il discute la problématique des relations chercheurs-terrains en sciences de gestion et montre que le statut de terrain est encore très flou, voire contradictoire, selon les recherches. En ce sens, il montre que le terme «terrain» se caractérise par une certaine ambiguiité sémantique en sciences de gestion et que le statut épistémologique du terrain reste relativement flou dans la mesure où il existe une grande variété d'utilisations. D'autre part, le 
parti pris épistémologique et méthodologique est de mener des recherches expérimentales, à visée transformative, en participant directement à des actions visant à modifier certaines caractéristiques du fonctionnement des organisations. Cette option épistémologique fondamentale donne donc au chercheur en gestion un rôle sociétal dans l'amélioration de l'efficacité des organisations. Cette approche transformative du champ phénoménologique observé facilite ainsi l'étude des différents stades de transformations et permet l'élaboration et la construction de théories et de méthodes dans le cadre d'un travail d'intention scientifique qui peut être orienté pour engendrer des progrès dans les connaissances. Enfin, il convient d'insister sur le fait que la création de nouvelles hypothèses nécessite de prendre du recul par rapport au terrain et requiert quasiment autant de travail que la présence effective dans les organisations. En définitive, la recherche-intervention apparaît comme une méthode de recherche scientifique légitime dans les épistémologies constructivistes (Le Moigne, 1995) qui postulent qu'il n'est de connaissances que des représentations artificielles construites intentionnellement et que l'on ne peut pas séparer l'observateur de l'observation.

\subsection{La recherche-action en PME: contexte d'intervention et éléments d'ordre méthodologique}

Dans cette section, notre objectif est d'identifier et de discuter plusieurs problèmes complexes et interactifs posés par l'analyse ethnographique de la PME. En ce sens, on abordera deux types de problèmes relatifs à l'analyse de la PME qui seront développés compte tenu de la problématique annoncée :

- le contexte d'intervention en PME : quelles spécificités propres à ce type d'investigation?

- le problème de l'identité du chercheur et la nature de ses relations avec le propriétaire-dirigeant : quels dispositifs d'analyse?

En tant que contexte d'intervention, la PME présente trois traits particuliers liés à la possibilité d'externaliser des services ou une partie de la production, à son système de gestion singulier et à l'influence du dirigeant sur toute la gestion de l'entreprise ainsi que dans ses relations avec l'intervenant-chercheur (Plane et Ferrer, 1997).

- La PME et l'externalisation de services de management. Si l'on s'attache à l'image désormais bien connue de la chaîne de valeur de Porter, les avantages de l'externalisation de services de management peuvent être importants pour la PME. Le dirigeant de PME peut donc se centrer sur les fonctions clés de sa chaîne de valeur, c'est-à-dire celles qui contribuent directement à l'avantage compétitif. Pour les autres fonctions, les avantages de la transaction l'emporteraient sur l'internalisation. Au total, le système 
de gestion propre à la PME conduit à un évitement de capacité. Et l'appel à des investigations externes peut apparaitre intéressante pour un dirigeant souhaitant bénéficier d'un effet de recadrage, c'est-à-dire d'une manière différente de voir ou de poser les problèmes. En fait, l'intervention d'un chercheur dans une PME traduit d'abord une démarche de l'entreprise qui accepte explicitement, voire attend, une remise en cause, une rupture quant à sa manière de traiter les problèmes.

- La PME : un système de gestion interpénétré. La PME est dotée de caractéristiques organisationnelles propres, de méthodes de gestion rudimentaires parfois, et d'une très forte intégration des objectifs de gestion. En ce sens, on observe peu de différenciation en grandes fonctions (marketing, ressources humaines, etc.) comme on les retrouve dans les grandes entreprises. Il en résulte une forte interpénétration des trois niveaux du système de gestion ainsi que des problèmes opérationnels. En conséquence, un problème donné pourrait s'analyser à partir des implications pour les différents sous-systèmes, ce qui lui confère une forte dimension stratégique. Pour maîtriser cette complexité, il faut tenir compte de la particularité de ce système de gestion. En effet, les problèmes abordés ne sont ni simples ni compliqués, mais complexes, c'est pourquoi leur résolution suppose une capacité de réflexion globale. L'intervenant-chercheur doit donc tenir compte du caractère systémique de son investigation, ce qui suppose des compétences d'expert en management, mais aussi et surtout de facilitateur ou de catalyseur (Schein, 1989) dans la mesure où il s'agit d'aider le dirigeant à découvrir de nouvelles pistes d'actions possibles.

- La gestion des relations chercheur-dirigeant. Un des principaux éléments descriptifs de la PME est la centralité, l'omniprésence et la responsabilité exclusive de son dirigeant dans toutes les décisions. De fait, la gestion de l'intervention en PME est spécifique même si l'on peut noter que l'activité de l'intervenant-chercheur se présente comme un ensemble d'opérations destinées à aider le dirigeant à percevoir, à comprendre et à agir à partir de faits ou d'événements qui existent ou surgissent dans son environnement. Néanmoins, le simple fait de demander de l'aide induit dès le départ une situation sociale déséquilibrée dans notre société où l'autonomie est particulièrement importante en PME. En effet, dans la mesure où le demandeur de prestations se place, dans l'échange social, en position de dépendance face à celui qui va l'aider, il se crée un déséquilibre et une vulnérabilité. Le déséquilibre et la vulnérabilité de la situation d'interaction nécessitent que le chercheur opère rapidement un rééquilibrage, sous peine de se faire rejeter par le dirigeant, pour lui avoir fait en quelque sorte perdre la face. En ce sens, il est important d'insister sur cette idée que le chercheur doit s'efforcer de s'inspirer autant que 
possible d'une approche générale de «l'acte d'aider», toujours dans une logique de facilitation et d'aide à la résolution de problèmes comme nous l'avons décrite précédemment.

Dans le cadre de plusieurs investigations conduites au sein de petites entreprises (Plane et Taverne, 1997), nous avons à plusieurs reprises constaté que si notre souhait dans le processus de recherche-action avait été d'occuper une position de neutralité dans l'organisation, cela aurait constitué une aspiration utopique. En effet, il semble que cette position soit difficile à tenir sur un terrain dans la mesure où le propriétaire-dirigeant et ses employés ont un besoin de représentation et d'identification mentale du chercheur. Par conséquent, ils le situent nécessairement à une place dans le système organisationnel à partir de leurs propres représentations mentales et lui confèrent un rôle social. Cependant, on a montré antérieurement que cette place est relativement contingente, voire instable, dans la mesure où le chercheur peut tenter de la construire à partir de ses propres actes dans le système organisationnel. Les discours et les comportements d'acteurs semblent ainsi conditionnés par la logique des processus d'interactions. À travers nos investigations au sein d'une petite entreprise de conseil en organisation, il est apparu progressivement aux autres acteurs que nous étions l'envoyé du dirigeant, dont nous étions probablement l'agent. Compte tenu de ces représentations mentales et de cette place, il était impensable pour ces personnes de nous confier toutes leurs opinions sur le fonctionnement de la société. On peut donc penser qu'il est capital de considérer les phénomènes de transfert du propriétaire-dirigeant et de contre-transfert. Il apparaît ainsi utile de détecter et de traiter les projections faites sur le chercheur par toutes les personnes, membres du terrain, quel que soit leur statut dans l'organisation (analyse en termes de transfert) ainsi que les projections faites par le chercheur sur les acteurs du terrain (analyse en termes de contre-transfert). Par conséquent, l'ampleur de ce phénomène constitue l'angoisse de l'observateur devant la richesse et la diversité de ses observations. Il semble que dès qu'il y a des processus d'interactions, cela produit des réactions d'acteurs qui peuvent fournir des enseignements suffisamment signifiants et solides pour un chercheur en quête de connaissances.

À propos de la position du chercheur en PME, on privilégiera la méthode interactive à visée transformative dont l'objectif est de transformer le fonctionnement de l'organisation observée. De plus, il est essentiel que les intervenants-chercheurs en PME optent pour une méthode d'investigation prenant en considération les dimensions systémique, interactive et holistique propres au système de gestion de la PME (Marchesnay, 1994). Cette méthode clinique implique que le processus de recherche-intervention représente le mode privilégié d'observation de phénomènes systémiques et interactifs. Enfin, compte tenu de la nature de la convention passée entre les chercheurs et le dirigeant de la PME, ces derniers ne peuvent pas être neutres, et à vrai dire ne souhaitent même pas l'être, puisque leur mission a pour finalité l'amélioration du fonctionnement interne de l'entreprise. Par ailleurs, il faut 
souligner que l'implication du chercheur en PME se caractérise par un effet de grossissement (Mahé de Boislandelle, 1996) dans la mesure où son rôle lui confère un poids hiérarchique considérable.

\section{Contribution de la démarche ethnométhodologique à l'analyse des PME: problèmes et éléments de méthodes}

Le contexte d'intervention en PME étant posé, il convient de s'intéresser aux spécificités ainsi qu'aux apports de la démarche ethnométhodologique à l'analyse de la PME. Dans cette perspective, nous traiterons successivement les deux questions suivantes posées par l'implication du chercheur en PME.

- Le problème de la dialectique immersion-distanciation de l'analyste vis-à-vis de l'objet étudié : quelles méthodes d'investigations mettre en œuvre?

- Le problème de l'implication du chercheur en PME : quels enjeux, quelle contribution?

\subsection{Le problème de la dialectique immersion-distanciation du chercheur en PME}

Nombre de chercheurs en sciences de gestion éprouvent des difficultés de dosage de la distance à adopter vis-à-vis de leur terrain d'investigations. Cette sous-section est donc consacrée à l'identification et à l'analyse de la dialectique immersiondistanciation du chercheur en management. On insistera sur l'idée que le chercheur en sciences de gestion doit pouvoir se ménager une position de recul et de distance critique lui permettant de rompre avec la réalité sensible afin d'enlever aux phénomènes observés leur caractère d'évidence qu'ils ont aux yeux des acteurs. On définira donc la notion de distance comme un espace qui empêche toute familiarité ; cette distance est liée, semble-t-il, à la position d'extériorité du chercheur et se caractérise notamment par une certaine retenue, voire une certaine prudence, dans la teneur de ses propos et dans les comportements adoptés dans les situations d'interaction (Plane, 1994, p. 301). Compte tenu de la définition suggérée, il semble souhaitable de l'illustrer à partir d'observations empiriques.

Lors des nombreux entretiens semi-directifs collectifs et individuels, nous avons perçu une distance relativement importante avec les interviewés dans la mesure où ils ne nous connaissaient pas. Par ailleurs, ces entretiens suscitent, à divers degrés, une certaine méfiance chez les acteurs. En ce sens, au sein d'une petite société de réalisation audiovisuelle, une personne a commis l'imprudence de citer un nom, ce qui a immédiatement provoqué une réaction de la part de ses collègues 
qui se sont adressés à elle de la manière suivante : «Pourquoi tu donnes des noms? » Cette observation indique que cette distance est particulièrement liée à la situation d'étranger au système organisationnel dans laquelle se trouve le chercheur. Il semble ainsi que cette position d'extériorité renforce l'idée qu'il est préférable de ne pas faire connaître des faits tabous que les acteurs préfèrent taire et que le chercheur risquerait de rendre explicites ou, pis, de les diffuser dans l'environnement de la PME. En effet, on a souvent exprimé des craintes et des inquiétudes quant au respect de l'anonymat de l'entreprise et de ses membres concernant notamment la diffusion des résultats obtenus à l'extérieur, même si un certain nombre de garanties ont été préalablement fournies. La prise de notes manuscrites contribue également au renforcement de ce phénomène et donc, à l'accroissement de la distanciation, car elle constitue une preuve physique des propos tenus. Ces propos seront, par ailleurs, soumis à un traitement qui fera l'objet d'une restitution dans l'organisation et d'une utilisation dans son environnement. Le style de questionnement du chercheur peut également accentuer le degré de cette distance. Au total, il apparaît que la semidirectivité peut conduire l'employé interviewé à esquisser des sous-thèmes tabous dans l'entreprise tels que la politique de rémunération, la stratégie du dirigeant ou, encore, son mode de management. La distance chercheur-acteurs ainsi développée présente manifestement cinq caractéristiques intrinsèques en PME.

- Cet espace lié à la position d'extériorité du chercheur est permanent dans la mesure où tout intervenant externe suscite toujours une certaine méfiance, voire une crainte, dans la mesure où l'on sait qu'il est susceptible de révéler des informations que les membres de l'entreprise préfèrent garder secrètes.

- Fondamentalement, le chercheur est un étranger dans l'entreprise provoquant ainsi des perturbations (méfiance, rétention d'informations, etc.).

- Le degré de distanciation adopté par certains acteurs est donc un phénomène construit de manière plus ou moins délibérée. Il semble en effet que cette prise de distance soit liée à des réflexes protecteurs. Le chercheur peut, cependant, tenter d'analyser les effets de ce degré de distanciation sur la teneur des propos tenus par les acteurs. Cette analyse au regard de la distance contribue ainsi à l'objectivation des informations extraites du terrain d'observation.

- L'espace existant entre un chercheur et les membres de l'entreprise peut également faire l'objet d'un pilotage par celui-ci en temps réel. En ce sens, il apparait possible pour un intervenant d'obtenir un degré de distanciation plus ou moins important en fonction des informations qu'il souhaite obtenir et des effets qu'il veut créer dans le cadre de ses prestations.

- Enfin, le degré de distanciation doit, semble-t-il, être suffisant, car une trop grande familiarité nuit à la nécessaire directivité dont peut faire preuve le 
chercheur pour stimuler certains employés afin de les conduire à la production d'actes de gestion.

Selon nous, cinq caractéristiques du degré de distanciation chercheur-acteurs précédemment décrites sont stimulantes pour le chercheur en gestion à deux conditions. D'une part, il est important que celui-ci soit conscient, par introspection, à la fois du degré de distanciation existant et de ses facteurs explicatifs. D'autre part, ces caractéristiques du système de distanciation peuvent constituer un moyen de traitement d'intention scientifique des informations extraites si le chercheur retranscrit les conditions dans lesquelles elles ont été obtenues.

Ainsi, nous avons acquis la conviction, lors de recherches en PME (Plane et Taverne, 1997), que le chercheur en management découvre progressivement l'entreprise par une immersion en profondeur dans celle-ci. Cependant, cette immersion suscite des phénomènes de rejet ainsi qu'une certaine méfiance des acteurs. C'est cette immersion d'un corps étranger au système organisationnel qui est à l'origine à la fois du degré de distanciation décrit, mais plus généralement, comme l'a montré Levi-Strauss (1950, p. 9), des transformations des comportements des personnes rencontrées. Dans le même sens, Avenier $(1989$, p. 205) note que ces interactions peuvent être érigées en instruments d'élaboration de connaissances. Cette conception de l'approche d'un terrain coïncide avec celle de différents chercheurs adoptant une approche constructiviste de la recherche. En effet, si la distance chercheurs-acteurs s'explique par l'immersion de corps étrangers au système organisationnel, ils ont souvent un regard gênant pour les acteurs ou, en tout cas, perturbant. Ces perturbations, au lieu de constituer des obstacles à la recherche, constituent des matériaux précieux tant pour accroître la qualité des prestations réalisées que pour améliorer le niveau de connaissances et donc de compétences des acteurs. Le degré de distanciation offre également la possibilité au chercheur de ne pas trop s'exposer aux risques de manipulations conscientes ou inconscientes de son travail par certains acteurs.

Compte tenu de cette dernière observation, il convient de montrer également les risques que présente un degré de distanciation trop élevé. Un étranger à une organisation est souvent considéré comme un intrus toujours suspecté, d'une part, de tenter de remettre en cause certains acquis et, $d$ 'autre part, de vouloir divulguer des informations que certains acteurs préféreraient ne pas exposer (Matheu, 1986, p. 86). En revanche, l'immersion des chercheurs dans l'organisation peut être trop superficielle, ce qui présente le principal risque de constituer une base d'informations insuffisamment signifiantes et, donc, de restituer des résultats erronés, altérant la légitimité de la recherche et la crédibilité des chercheurs. Enfin, un degré de distanciation trop important peut constituer un obstacle au développement de la nécessaire connivence entre les acteurs de l'organisation et le chercheur en gestion.

Les risques que l'on vient d'exposer nécessitent d'être relativisés à partir des atouts que comporte une telle immersion dans l'organisation; celle-ci présente la 
caractéristique d'être une quasi-internalisation. Cependant, on insistera sur l'idée qu'une démarche clinique recèle plusieurs avantages décisifs. En premier lieu, la recherche-intervention est une action de longue durée (variant de plusieurs mois à quelques années) réalisée en profondeur, car elle nécessite de multiples rencontres avec les acteurs dans le cadre d'entretiens et de prestations en management approfondies. En second lieu, l'action développée par les chercheurs s'inscrit généralement dans la stratégie de l'entreprise, au sens de Marchesnay (1993, p. 167 et s.), c'est-àdire qu'elle s'appuie notamment sur les buts du propriétaire-dirigeant de la PME. Cette dimension stratégique de la démarche contribue à l'obtention d'une collaboration étroite du dirigeant dans la mesure où les enjeux sont relativement forts puisque la démarche s'appuie sur des actions de transformation de l'entreprise (Plane et Ferrer, 1997). Par ailleurs, la quasi-internalisation du chercheur en gestion présente l'avantage décisif de faciliter la découverte de connaissances nouvelles, comme le note Moisdon (1984, p. 61 et s.), issues de multiples interactions. On joindra ce point de vue sur l'opportunité de produire de nouvelles connaissances par interactivité (Plane, 1994) à la condition d'une conscience accrue par le chercheur de la portée du triptyque «actes produits/impacts engendrés/effets obtenus et observables ».

Ces trois avantages décisifs étant posés, il convient de ne pas occulter les risques qui peuvent être liés à une telle immersion. Dans cette perspective, on distinguera trois risques relatifs à une trop forte immersion des chercheurs. Premièrement, il semble que le premier de ces risques soit la manipulation du chercheur par les acteurs de l'entreprise. Deuxièmement, le chercheur sur son terrain d'observation peut manquer de lucidité et de visibilité par une trop forte absorption du terrain. Troisièmement, on peut penser qu'il existe également un risque lié à une insuffisance de transformations produites dans l'entreprise. En effet, on soutient la thèse selon laquelle le processus d'intervention en management conduit au développement d'actes de gestions introspectifs et conceptifs ayant des effets immédiats et différés sur la performance de l'entreprise. Autrement dit, l'intervenant développe chez les acteurs une capacité nouvelle d'introspection et de conceptualisation qui est à la source de la performance (Plane, 1994, p. 568 et s.). Ces transformations doivent être suffisamment significatives dans l'entreprise. Si ce n'est pas le cas, à cause d'une trop forte absorption du chercheur par le terrain, sa légitimité risque d'être fortement remise en cause au sein de l'organisation. Ce dernier risque est probablement le plus conséquent.

\subsection{Contributions de la démarche ethnométhodologique à l'analyse de la PME}

À travers l'analyse des observations et des interactions produites au sein de trois PME et d'une recherche conduite auprès des dirigeants d'organismes de formation 
professionnelle de petite taille ${ }^{2}$, nous avons pu identifier quatre relations ambivalentes particulièrement intensifiées lorsqu'un chercheur est impliqué en PME.

\section{Le rapport entre le formel et l'informel}

En PME, le chercheur observe une prédominance des processus dans le mode de fonctionnement. L'absence de procédures, de planification et de programmation stratégique (Marchesnay, 1993) conduit le chercheur à travailler avec les acteurs sur des modes de régulation et d'actions processuels. Ce phénomène complexe conduit à rendre lisibles puis visibles des actes de gestion cachés. Toutefois, on peut souligner le fait que l'action du chercheur peut conduire, à partir d'un arbitrage, à l'accroissement du degré de formalisation. L'absence de règles formelles, de procédures ou de normes peut parfois être la cause de dysfonctionnements propres à la PME. Le rôle de l'intervenant-chercheur est alors de convaincre le dirigeant de la PME de l'utilité de mettre en place une plus grande formalisation des actes de gestion. La nécessité d'une plus grande formalisation au sein d'une PME semble s'affirmer dès lors que le propriétaire-dirigeant s'appuie sur des prestations de conseil en organisation ou en management. Dans ce cas de figure, le dirigeant souhaite progressivement passer d'une logique entrepreneuriale à une logique managériale dans la mesure où l'un des objectifs est de planifier et de programmer les activités. Au total, la question de la contribution d'un intervenant en management au développement d'une petite entreprise peut être traitée à partir de l'analyse de l'évolution du rapport informel et formel dans la structure.

\section{Le rapport entre le visible et le caché}

La démarche de recherche interactive, telle qu'elle a été exposée précédemment, suppose une certaine connivence entre l'entrepreneur et le chercheur. Dans cette optique, on peut penser que ce dernier va inévitablement découvrir des faits tabous ou des phénomènes implicites que nombre d'acteurs préfèrent ignorer. En ce sens, l'activité du chercheur n'est pas simple dans la mesure où il peut relever, par exemple, un caractère excessivement autocratique du dirigeant malgré des intentions louables. Dans ce cas, l'action du chercheur peut contribuer à une certaine dépersonnalisation du mode de gestion de l'entreprise alors que le dirigeant peut, par exemple, décider de déléguer davantage. Par ailleurs, la mise en évidence de nombreux coûts cachés (Savall et Zardet, 1987) dans la structure peut constituer un véritable catalyseur pour le propriétaire-dirigeant qui peut ainsi prendre la décision de renforcer la logique

2. Recherche financée par l'observatoire régional de l'emploi, des qualifications et des formations du Languedoc-Roussillon (OREQF) sur l'analyse du comportement stratégique des dirigeants d'organismes de formation professionnelle de petite taille (de janvier à décembre 1996). 
de contrôle des coûts. L'intervenant-chercheur provoque des perturbations chez les acteurs en explicitant des informations nouvelles qui remettent en cause certaines pratiques de gestion de l'entreprise (comme, par exemple, les pratiques de délégation concertée en petite entreprise). Ces nouvelles informations rendent explicite ce qui est implicite, ce qui revient à formaliser des informations implicites qu'une partie des acteurs préfèrent taire ou tout au moins ne pas rendre publiques (Plane, 1994 et 1996b). Pour ce faire, le chercheur met en œuvre des techniques de déstabilisation en bousculant les usages de l'entreprise, c'est-à-dire en produisant des actes non conformes aux pratiques courantes ou usuelles des acteurs. Ce non-conformisme constitue une source de perturbation et permet de parler d'un véritable pouvoir interactif du chercheur si l'on admet l'asymétrie des relations avec les acteurs. Cependant, il est important de souligner la nécessité pour l'intervenant de s'appuyer sur des techniques de stabilisation des acteurs afin d'éviter toute éviction de l'organisation discutée précédemment (même si 1'on considère l'éviction comme un phénomène ontologique scientifiquement intéressant). La stabilisation des comportements humains suppose que les informations activantes soient fondées sur une authenticité incontestable, ce qui semble nécessiter une base d'information de qualité suffisante.

\section{Le rapport entre le dire et le faire}

Comme on vient de le mentionner, le rapport entre le dit et le non-dit dans les organisations est problématique pour tout intervenant. En fait, différentes observations semblent indiquer que les causes profondes des problèmes stratégiques rencontrés par l'entrepreneur sont rarement évoquées spontanément lors des entretiens comme, par exemple, l'organisation personnelle, le style de direction ou, encore, les méthodes de travail. Une fois de plus, l'intervention dans le cadre d'une PME peut s'avérer particulièrement perturbatrice dans la mesure où le chercheur devra faire face à une part de non-dit plus importante que dans les grandes organisations. Dans le cadre de recherches antérieures, nous avons eu l'occasion de montrer que l'intention stratégique ne constitue pas nécessairement un gage de mise en œuvre (Plane, 1994). En PME, il apparaît que la place de l'oralité est très forte. L'attraction des dirigeants de PME pour les informations verbales a été maintes fois mise en évidence (Fallery, 1983). «La préférence des chefs d'entreprise pour la communication orale se traduit par le recours à des processus mentaux de mémorisation et de traitement des informations de développement. Il est dès lors inévitable que la personnalité et les structures qu'ils mettent en place exercent des influences majeures sur les démarches de recherche et d'information des entreprises. » (Philippe, 1990) Cette prédominance de la parole constitue à la fois une richesse pour un observateur, mais également une source de difficulté supplémentaire dans la mesure où ce que l'on pourrait appeler «l'agir communiquant » ne semble pas toujours se présenter. Le rôle de l'intervenant sera alors de faire la part entre l'intention déclarée et l'action effective. 


\section{Le rapport entre l'implicite et l'explicite}

À partir de la mise en place d'analyseurs plus ou moins puissants (diagnostic, analyse de la gestion du temps, carte cognitive, etc.), le chercheur est très souvent conduit à expliciter l'implicite. Pour ce faire, on peut penser qu'il va collecter, organiser puis restituer des informations socialisées au sein de la structure, mais largement implicites. Par exemple, il est généralement admis qu'en PME, l'attention du dirigeant est souvent focalisé sur les problèmes urgents. Selon Mahé de Boislandelle (1996), «L'effet de grossissement tend à contracter les horizons de calcul ». Dans ces conditions, un travail sur l'urgence en PME conduit à identifier des actes de gestion courante réalisés dans l'urgence alors que leur valeur ajoutée semble très faible. En d'autres termes, cette explicitation permet à l'entrepreneur de s'interroger sur le caractère excessivement centralisé des modes de gestion de son entreprise et sur la possibilité de déléguer des activités à faible valeur ajoutée.

En définitive, il convient d'insister sur les apports spécifiques et expérimentés de la démarche ethnométhodologique en PME. Fondamentalement, la démarche présente un caractère fortement interactif dès lors que les investigations s'appuient sur des grilles d'analyse ou sur des outils de gestion interactifs comme, par exemple, une grille d'analyse de la gestion du temps du dirigeant ou une cartographie des compétences. Ces instruments de gestion coproduits avec le propriétaire-dirigeant sont relativement structurants et permettent de construire une relation de travail professionnelle. Cependant, il est important de relever que les informations recueillies, traitées puis restituées par le chercheur doivent être suffisamment authentiques et pertinentes pour le dirigeant pour constituer un gage de crédibilité et de professionnalisme pour l'intervenant-chercheur. Ainsi, l'analyse des compétences au sein de la PME peut fournir au dirigeant une représentation instructive de l'état de son potentiel humain pouvant être particulièrement utile pour l'action stratégique. Par ailleurs, il convient de ne pas perdre de vue le caractère immatériel des prestations réalisées à partir de cette approche de l'entreprise. En effet, il semble que l'équipe de recherche devra légitimer son action en s'efforçant de produire des résultats intermédiaires. Ainsi, on peut penser que l'analyse de la gestion du temps du dirigeant doit rapidement conduire à des résolutions effectives comme, par exemple, l'accroissement du temps de travail consacré à l'activité commerciale ou à la communication externe. Dans le but de développer l'efficacité de ce type d'approche, il s'agit d'impliquer le plus grand nombre possible d'acteurs dans la démarche, entre autres, à partir d'un groupe de travail. Ce dispositif présente l'avantage d'associer le dirigeant à ses salariés, ce qui peut le conduire à leur déléguer des missions stimulantes. Par exemple, deux salariés pourraient réfléchir sur la mise en place de règles et de procédures de fonctionnement ou sur des indicateurs visant à enrichir le tableau de bord de pilotage de l'entreprise qui est probablement encore largement informel. Enfin, il convient de ne pas perdre de vue la nécessité pour l'intervenant-chercheur d'aider le dirigeant «à s'aider» sans pour autant lui faire 
perdre la face, car on sait que cette forme d'investigation induit inévitablement un déséquilibre entre l'offreur et le demandeur.

\section{Conclusion}

En guise de conclusion, on peut soutenir que les problèmes théoriques et méthodologiques posés par cette approche ethnographique - reposant largement sur une conception ethnométhodologique d'analyse des discours et des pratiques au sein de PME - paraissent stratégiques puisqu'ils peuvent contribuer à l'amélioration des connaissances dans ce domaine. Finalement, il semble que l'intervention dans le cadre d'une PME présente plusieurs particularités compte tenu du fait que la PME constitue un terrain d'investigation particulier. Mais le paradoxe est précisément que l'intervention en PME peut déboucher sur un processus dénaturant, au sens où le définit Torrès (1997). En effet, le chercheur tend à adopter un comportement radicalement opposé aux comportements habituels dans une PME : il aura tendance à expliciter l'implicite, à formaliser l'informel, à retranscrire par écrit ce qui n'était jusqu'alors que du domaine de l'oral, à procéduraliser le processuel, etc., c'est-àdire à adopter des actions à visée transformative qui vont à l'encontre de la spécificité de la PME. En définitive, la thèse que nous défendons est que l'approche ethnométhodologique peut faciliter l'introduction de comportements et de modes de gestion managériaux au sein de la PME, c'est-à-dire dans un contexte entrepreneurial. Enfin, nous tenons à signaler que nous souhaitions montrer dans quelle mesure la recherche-intervention, fondée sur une approche ethnométhodologique des pratiques de gestion des PME, peut contribuer au développement de recherches d'intentions scientifiques et d'actions à visée transformative dans un domaine encore trop délaissé.

\section{Bibliographie}

AVENIER, M. J. (1989), « Méthodes de terrain et recherche en management stratégique », Revue Économies et Sociétés, Série Sciences de gestion, $\mathrm{n}^{\circ}$ 14, p. 199-218.

Bizaguet, A. (1991), Les petites et moyennes entreprises, Paris, Presses universitaires de France, coll. «Que sais-je ?», n 2642,127 pages.

BOUCHIKHI, A. (1988), Éléments d'une approche constructiviste des structures organisationnelles. Cas de la structuration d'un champ de la micro-informatique à la RATP, Thèse pour le doctorat en méthodes scientifiques de gestion, Université Paris IXDauphine, 251 pages.

LES CAHIERS DE CARGÈSE-CNRS (1991), Entreprises et chercheurs à la recherche d'un partenariat, Paris, L'Harmattan, coll. «Logiques sociales », 190 pages.

Coulon, A. (1993), L'ethnométhodologie, Paris, Presses universitaires de France, coll. «Que sais-je ?», n 2393,127 pages. 
D'AMBoISE, G. (1993), «Empirical research on SME's : the past ten years in Canada », Journal of Small Business \& Entrepreneurship, vol. 10, nº 2, p. 2-12.

Devereux, G. (1980), De l'angoisse à la méthode dans les sciences du comportement, Paris, Flammarion, 474 pages.

FALLERY, B. (1983), «Un système d'information du dirigeant pour les PME», Revue française de gestion, novembre-décembre, p. 70-76.

GARFINKEL, A. (1967), Studies in Ethnomethodology, Englewood Cliffs, NJ, Prentice-Hall, 152 pages.

GIRIN, J. (1986), « L'objectivation des données subjectives. Éléments pour une théorie du dispositif dans la recherche interactive », Communication au colloque FNEGEISEOR des 18 et 19 novembre à Lyon, Actes publiés par l'ISEOR, p. 170-186.

GIRIN, J. (1990), «L'analyse empirique des situations de gestion : éléments de théorie et de méthode », dans Epistémologies et Sciences de gestion, Paris, Economica, p. 141-182.

GREPME (1994), Les PME. Bilan et perspectives, ouvrage collectif coordonné par P.A. Julien, Paris, Economica, 437 pages.

Julien, P.A et M. Marchesnay (1987), La petite entreprise. Principes d'économie et de gestion, Paris, Vuibert, 288 pages.

Julien, P.A. et M. MARChESNAY (1996), L'entrepreneuriat, Paris, Economica poche, 112 pages.

Julien, P.A. et M. MARChESNAy (1997), Économie et stratégie industrielles, Paris, Economica poche, 112 pages.

LARONCHE, M. (1997), «Les multinationales, porte-avions des PME à l'exportation », Le Monde Économie du 2 septembre, p. 7.

LE MoIGnE, J. L. (1995), Les épistémologies constructivistes, Paris, Presses universitaires de France, coll. «Que sais-je ?», n 2969, 127 pages.

LÉVI-STRAUSS, C. (1950), «Introduction à l'œuvre de Marcel Mauss », dans M. Mauss, Sociologie et anthropologie, Paris, Presses universitaires de France, 482 pages.

MAHÉ De Boislandelle, H. (1996), «L'effet de grossissement chez le dirigeant de PME : ses incidences sur le plan du management des hommes et de la GRH », Communication au $3^{\mathrm{e}}$ congrès international francophone PME (CIFPME 96), Trois-Rivières, Québec, p. 101-115.

MARCHESNAY, M. (1985), «Quelques propositions méthodologiques appropriées aux sciences de gestion », Communication au colloque FNEGE-ISEOR des 13 et 14 novembre à Lyon, Actes publiés par l'ISEOR, 285 pages, p. 53-61.

MARCHESNAY, M. (1993), «PME, stratégie et recherche», Revue française de gestion, $\mathrm{n}^{\circ} 95$, p. $70-76$.

MARCHESNAY, M. (1994), «Le management stratégique», dans l'ouvrage collectif du GREPME, Les PME. Bilan et perspectives, Paris, Economica, 437 pages, p. 133-162.

MAtheu, M. (1986), «La familiarité distante », Revue Gérer et Comprendre, Annales des Mines, p. 81-94. 
MoISDON, J. C. (1984), «Recherche en gestion et intervention », Revue française de gestion, $\mathrm{n}^{\text {os }} 47-48$, p. 61-73.

MORIN, P. (1987), «L'intervention dans l'entreprise et le développement des organisations », Traité de Psychologie du travail, Paris, Presses universitaires de France, 826 pages, p. 77-101.

Nobre, T. (1995), «Le processus de structuration dans la petite entreprise. Une étude exploratoire», Revue Internationale PME, vol. 8, $\mathrm{n}^{\circ}$ 2, p. 203-238.

PHILIPPE, J. (1990), «Information et milieu économique : des ressources à mobiliser», dans Stratégies internationales des PME, Paris, Economica, 263 pages.

PlaNe, J. M. (1994), Contribution de l'intervention en management au développement de l'entreprise, Thèse pour le doctorat ès sciences de gestion, décembre, Université Lumière Lyon II, 741 pages +336 pages annexes.

PlANE, J. M. (1996a), « Pour une approche ethnométhodologique de la petite entreprise », Actes du $3^{e}$ Congrès international francophone de la PME, Université du Québec à Trois-Rivières, tome 2, p. 1106-1117.

Plane, J. M. (1996b), «Pouvoir interactif des intervenants en management stratégique », Revue Sciences de la Société, $\mathrm{n}^{\circ}$ 38, p. 111-127.

Plane J. M. et M. I. Ferrer (1997), «Le conseil à la PME»dans Gestion de la PME/ $P M I$, ouvrage collectif coordonné par M. Marchesnay et C. Fourcade, Paris, Nathan, 317 pages, p. 125-133.

Plane, J. M. et D. TAVERnE (1997), «Stratégies de repositionnement des organismes de formation », Revue Interface en Languedoc-Roussillon, Ministère du Travail et des Affaires sociales, $\mathrm{n}^{\circ}$ 6, p. 43-47.

SAVALl, H. (1985), «Où va la recherche en Sciences de Gestion?», Revue française de gestion, $\mathrm{n}^{\text {os }} 53-54$, p. 242-253.

SAVAll, H. (1989), «Point de vue : Professeur-consultant. Le bilan d'une expérience», Revue française de gestion, $\mathrm{n}^{\circ}$ 76, p. 93-105.

SAVALL, H. et V. ZARdeT (1987), « Maîtriser les coûts et les performances cachés », Economica, 351 pages.

SCHEIN, E.H. (1987), Process Consultation. Lessons for Managers and Consultants, Reading, Mass., Addition-Wesley, OD series, 212 pages.

TORRÈs, O. (1996), « Pour une approche contingente de la spécificité de la PME », Actes $d u 3^{e}$ Congrès international francophone de la PME, Université du Québec à TroisRivières, tome 2, p. 789-808.

TORRÈs, O. (1997), Pour une approche critique de la spécificité de gestion de la PME: application au cas de la globalisation, Thèse de doctorat de l'Université de Montpellier I, 352 pages.

YANAT, Z. (1992), «L'ethnométhodologie, démarche pour un audit social stratégique efficace», Revue de Gestion des ressources humaines, $\mathrm{n}^{\circ} 3$, p. 23-32. 